\title{
Evaluación de hábitos de alimentación, actividad física y estado nutricional en estudiantes de 4 a 13 años de la comuna de Llanquihue, Chile
}

\author{
Evaluation of food habits, physical activity and nutritional status in students \\ 4 to 13 years of age in Llanquihue, Chile
}

\begin{abstract}
RESUMEN
Datos generales de la Junta de Auxilio Escolar y Becas (JUNAEB) señalan la Región de Los Lagos como la segunda con mayor prevalencia de sobrepeso y obesidad en Chile. El objetivo de este estudio fue evaluar los hábitos de alimentación y actividad física de estudiantes de la Comuna de Llanquihue, Región de Los Lagos. En una sub-muestra de 181 estudiantes de entre 4 a 13 años de edad se evaluaron los hábitos de alimentación y actividad física; y a la muestra total $(n=1045)$ se les realizó una evaluación del estado nutricional. El estado nutricional se clasificó como normal, sobrepeso y obesidad y fue determinada según estándares y normativas del Ministerio de Salud. Los resultados señalan que la gran mayoría de los estudiantes encuestados almorzaron en el colegio; consumieron alimentos altos en azúcar refinada, productos envasados y comida rápida al menos 1 vez a la semana. El 58,5\% de los encuestados no realizó actividad física extra-programática. Finalmente el 29,4\% de la totalidad de la muestra presentó sobrepeso y el 26,2\% obesidad. Se puede concluir que los hábitos alimentarios y de actividad física no contribuyen a revertir las cifras de malnutrición por exceso detectadas en esta investigación. Palabras clave: Nutrición; Llanquihue; Obesidad; Prevalencia; Sobrepeso.
\end{abstract}

\footnotetext{
ABSTRACT

General data from the Junta Nacional de Auxilio Escolar y Becas (National School Grant and Scholarship Agency), indicate that the Los Lagos region ranks second in Chile for highest prevalence of overweight and obesity. The objective of this study was to evaluate eating habits and physical activity in Llanquihue, Los Lagos region. We evaluated eating habits and physical activity among 181 students between 4 and 13 years of age who were part of a larger sample $(n=1045)$ in which nutritional status was determined. Nutritional status was classified as normal, overweight, and obese, and was determined according to standards and regulations of the Ministry of Health. The results indicate that a majority of the students surveyed ate lunch at school, consumed foods high in refined sugar, packaged processed products, and junk food at least once a week. 58.6\% of respondents did
}

Corina Flores Calderón ${ }^{1}$, Gabriela Entrala Neira ${ }^{1}$, Jennyfer Flórez-Méndez ${ }^{1 *}$.

1. Centro de Estudios CEUS Llanquihue, Universidad de Santiago de Chile Vicente Pérez Rosales 709, Llanquihue, Chile.

*Dirigir correspondencia a: Jennyfer Flórez-Méndez, Centro de Estudios CEUS Llanquihue. Universidad de Santiago de Chile, Av. Vicente Pérez Rosales 709, Llanquihue, Región de los Lagos, Chile. Email: florez.jennyfer@gmail.com

not engage in extra-programmatic physical activity. Finally, $29.4 \%$ of the total sample were overweight and $26.2 \%$ were obese. It can be concluded that eating and physical activity habits do not contribute to reversing the excess malnutrition figures detected in this study.

Key words: Nutrition; Llanquihue; Obesity; Overweight Prevalence.

\section{INTRODUCCIÓN}

Según la OMS, el sobrepeso y la obesidad (SO) se definen como una acumulación anormal o excesiva de grasa que puede ser perjudicial para la salud, una forma simple de medir la obesidad es el índice de masa corporal (IMC) ${ }^{1}$, además, influyen diversos factores; la globalización, cultura, condición 
económica, educación, urbanización, entornos político y social $^{2}$, además, la obesidad incrementa la posibilidad de sufrir durante la infancia; baja autoestima, bajo rendimiento académico, ausentismo escolar y riesgos de salud ${ }^{3}$.

Existen muchos obstáculos para enfrentar la reducción del SO infantil, según la OMS, el principal impedimento es la falta de compromiso político y gubernamental ${ }^{4}$, las medidas utilizadas se concentran en: 1) Orientadas a promover la alimentación saludable y menos calórica, y 2) Aquellas destinadas a incentivar la actividad física ${ }^{5}$.

Chile a lo largo de su historia ha enfrentado problemas sociales y de salud como la desnutrición y la alta mortalidad infantil, los cuales han sido superados de manera exitosa y han hecho de Chile un referente internacional en la materia ${ }^{6}$, gracias a la implementación de estas medidas, en nuestro país la desnutrición se ha convertido en una leyenda ${ }^{7}$, no obstante, en la actualidad el país se encuentra dentro de aquellos con mayores tasas $\mathrm{SO}$ infantil, alcanzando un 9,3\% para la población menor de 5 años de edad, superando el promedio de América Latina y el Caribe $(7 \%)^{8}$. La obesidad es una condición crónica de alta prevalencia en Chile y en la mayoría de los países del mundo ${ }^{9}$, por su parte la obesidad infantil y adolescente no es un fenómeno transitorio ${ }^{10}$, la obesidad infantil es un claro predictor de la obesidad adulta ${ }^{11}$. El alarmante crecimiento de la prevalencia de obesidad en nuestra sociedad nos indica que debemos estar muy alerta, para el diagnóstico precoz de esta patología de alta repercusión en la salud de las personas y en los costos asociados a ella, ${ }^{9,12}$. Se considera que desde la primera infancia los niños obesos sin ningún tratamiento se mantendrán en esa condición hasta la adolescencia y edad adulta desarrollando diversas patologías ${ }^{13}$. En la actualidad existen múltiples políticas públicas enfocadas a la nutrición infantil ${ }^{6}$, una de ellas es la ley 20.606 cuyo objetivo es proteger la salud de los chilenos, en especial la población infantil, entregando más información de los alimentos, para así lograr una mejor selección ${ }^{14}$.

Según la Encuesta Nacional de Salud de Chile 20162017, la obesidad afecta uno de cada cuatro niños, con alta prevalencia de obesidad en las escuelas municipales $(30 \%)^{15}$. El mapa nutricional de la JUNAEB de 2018 declara que a nivel nacional, existen altos índices de obesidad infantil, desde el primer nivel de transición a quinto año básico superando el 23\%, en el total de los cursos del rango ${ }^{16}$. En un estudio realizado a colegios de Santiago de Chile, se dio a conocer que existe una distorsión de la imagen corporal de los hijos, se observó que las madres de niños con sobrepeso solo el $17,4 \%$ coinciden con la imagen corporal que le corresponde ${ }^{17}$. Si se analiza el sector y condición socioeconómica, según estudio realizado entre los años 2009 y 2013 a estudiantes chilenos de primero básico se observa que existe un incremento de $\mathrm{SO}$ en niños más vulnerables de sectores rurales de un 16,6 a un $24,3 \%{ }^{18}$. Según JUNAEB (2018), la región de los Lagos, resultó ser la segunda región con mayor prevalencia de obesidad en estudiantes tanto del segundo nivel de transición como de quinto básico ${ }^{17}$. Los hábitos dietarios adecuados presentes desde etapas tempranas de la vida, contribuirían a garantizar la salud en la edad adulta.
No es fácil prevenir la obesidad infantil, ya que se requiere de cambios de estilo de vida y del compromiso de todo el grupo familiar ${ }^{19}$. Es de suma importancia la valoración de la función de la familia como una institución en la que se aprenden, socializan conductas, en este caso las de tipo alimentarias y de actividad física ${ }^{20}$. Actualmente existe evidencia suficientemente robusta que respalda la efectividad de las intervenciones orientadas a promover ambientes escolares saludables ${ }^{21}$, diversas revisiones en literatura científica han demostrado que las intervenciones de múltiples componentes, donde se incluye dieta, actividad física y cambios conductuales, resultan beneficiosas para conseguir pequeñas reducciones del IMC y peso en los niños en el corto plazo ${ }^{22}$. Por lo anterior, este estudio tiene como objetivo evaluar los hábitos de alimentación, actividad física en una submuestra que comprende 181 estudiantes y el estado nutricional de una muestra de 1.045 estudiantes, en ambos casos de 4 a 13 años de edad de la comuna de Llanquihue mediante encuesta.

\section{SUJETOS Y METODO Diseño del estudio}

La investigación corresponde a un estudio tipo descriptivo, con mediciones y registros realizados durante los meses de marzo a mayo de 2018 en la comuna de Llanquihue, Región de Los Lagos en Chile.

\section{Sujetos}

Este estudio abarcó una submuestra con 181 estudiantes de prebásica y básica de 10 establecimientos educacionales seleccionados de manera aleatoria con clasificación antropométrica normal, con sobrepeso y obesidad para los ítems de alimentación, frecuencia alimentaria y actividad física, esta encuesta aplicada tenía ítem de estilos de vida saludable, como la higiene, y modo de realizar la alimentación, también incluye una encuesta de frecuencia de consumo de alimentos cotidianos, saludables y no saludables, en cuanto a la actividad física se buscó encontrar una frecuencia de realización, que los motivó a realizar deporte, para posteriormente ampliar la muestra a fin evaluar el estado nutricional de 1.045 estudiantes de 5 establecimientos educacionales en la comuna de Llanquihue.

\section{Alimentación, frecuencia alimentaria y actividad física}

Los ítems evaluados fueron tres; aspectos nutricionales, de frecuencia alimentaria y de actividad física. Se utilizó técnica de recolección de datos estandarizada, por lo que en todos los casos el instrumento para la medición fue una encuesta basada en otros estudios dirigidos a población con similares características, la cual se aplicó bajo la modalidad de entrevista ${ }^{23,24}$.

\section{Evaluación del estado nutricional}

El universo de estudio lo comprendían estudiantes de enseñanza prebásica y básica, representados por un total de 1.045 estudiantes de cinco establecimientos educacionales de la comuna de Llanquihue, entre ellos cuatro municipales y uno particular subvencionado. Los criterios de exclusión fueron 
niños con alguna patología que lo impida formar parte de la muestra o niños con algún tipo de incapacidad física.

El estudio consistió en una Evaluación Nutricional Antropométrica para lo cual se realizó medición de peso y talla mediante una balanza mecánica con tallímetro incorporado, de la marca SECAÒ, calibrados de manera previa a las evaluaciones. Donde, a partir del peso y de la talla se calculó IMC para estudiantes mayores de 5 años y el puntaje $z$ de peso para la talla en estudiantes de 5 años de edad o menor según norma vigente de MINSAL. El procedimiento fue realizado por profesionales locales del Centro de salud familiar CESFAM Los Volcanes de la comuna de Llanquihue, las cuales acudieron a cada establecimiento para realizar la evaluación nutricional según sus parámetros internos establecidos para dichos fines.

La interpretación de los datos, se llevó a cabo por profesionales de la Universidad de Santiago de Chile, donde se estableció lo siguiente: Los niños, niñas y adolescentes desde los 5 años a 19 años fueron evaluados por IMC para la edad, según los estándares establecido por la norma para evaluación nutricional de MINSAL, las tablas registradas en esta norma informan el año y mes cumplido, por lo tanto, se utilizó un promedio por cada edad, dando los z-scores: Bajo peso: menos 0.1 del $-1 \mathrm{DE}$; Normal: de -1 a $1 \mathrm{DE}$ (menos 0.1); Sobre peso: de 1 DE hasta 2 DE (menos 0.1); Obesidad: 2 DE para arriba en categorías.

\section{Consideraciones éticas}

Las consideraciones éticas fueron basadas en los principios del tratado de Helsinki ${ }^{25}$ por lo que no se contó previamente con la aprobación de un comité de ética ya que tal como lo establece este tratado se dio la importancia de contar con el consentimiento de cada padre, madre y/o tutor legal quienes mediante un consentimiento informado aceptaron que su hijo participaría en el estudio, al igual que se contó con el asentimiento del sujeto en edad infantil y del mismo modo cada institución educativa apoyo y revisó cada consentimiento informado. Todas mediciones de peso y talla de los estudiantes fueron realizadas bajo supervisión de profesionales de la salud de CESFAM Los Volcanes de la comuna de Llanquihue.

\section{Plan de análisis}

Los datos fueron analizados con Statgrphics ${ }^{\circledR}$ Centurion, ver. 17 (Statpoint technologies, Inc), se utilizó el coeficiente de alfa de Cronbach $^{26}$ para evaluar la confiabilidad de la encuesta y el test de comparación de medianas para comprobar normalidad de los datos. Para variables categóricas se utilizó estadística no paramétrica. Para el análisis se utilizó alfa de Cronbach que estima la confiabilidad o la consistencia de un conjunto de variables y como regla general, se considera que un alfa igual a 0,7 o mayor representa un conjunto confiable de variables.

\section{RESULTADOS}

La tabla 1 muestra que los resultados del el alfa de Cronbach fueron mayor a 0,7 para cada ítem-escala lo cual establece la consistencia interna de las preguntas seleccionadas para el cuestionario aplicado en la encuesta. Los resultados obtenidos en la encuesta relacionada con el ítem nutrición llevada a cabo con 181 estudiantes de

Tabla 1. Consistencia de las preguntas de los distintos; Nutrición, Frecuencia alimentaria y actividad física en cursos de estudiantes de enseñanza básica de 10 establecimientos educacionales de la comuna de Llanquihue ( $\mathrm{n}=181$ ).

\section{Alfa Cronbach}

Ítem Nutrición

1. ¿Dónde realizas el almuerzo?

2. ¿Existen colaciones saludables entre comidas?

Ítem Frecuencia Alimentaria

1. ¿Con que frecuencia del 1 al 7 (días de la semana) consumes algo con azúcar refinada? (queque, tortas, galletas, kuchen, etc).

2. ¿Con que frecuencia del 1 al 7 (días de la semana) consumes productos envasados? (snack de bolsa, papas fritas, ramitas, etc.)?

3. ¿Con que frecuencia del 1 al 7 (días de la semana) consumes comida chatarra? (completos, churrascos, pizza, sopaipillas, etc.)?

Ítem Actividad física

1. ¿Realizas alguna actividad extraprogramática, o taller relacionado con el deporte? 
enseñanza básica de 10 establecimientos educacionales de la comuna de Llanquihue, mostró que la mayor parte de los niños encuestados almuerzan en el colegio y afirman consumir colaciones saludables entre comidas. Para el ítem frecuencia alimentaria, los resultados se muestran en la figura 1, donde se puede observar que la mayor parte de los estudiantes consumen azúcar refinada $(\mathrm{A})$, alimentos envasados y comida chatarra (B) al menos 1 vez a la semana.

La tabla 2 muestra los parámetros estadísticos y límites de los intervalos de confianza de comparación de medianas para menores y mayores a 5 años, de 10 establecimientos educacionales de la comuna de Llanquihue.

La figura 2 muestra el diagnóstico del estado nutricional de estudiantes mayores a 5 años (Figura 2A) de 5 establecimientos educacionales de la comuna de Llanquihue $(n=919)$ donde se aprecia un porcentaje importante que los estudiantes que participaron en este estudio se encuentra con sobrepeso y obesidad, con valores de 31,3 y $29,1 \%$, respectivamente y el diagnóstico del estado nutricional de estudiantes menores a 5 años (Figura 2B) de 5 establecimientos educacionales de la comuna de Llanquihue $(n=126)$ donde se aprecia que el porcentaje se encuentra con sobrepeso y obesidad menor que en el grupo de mayores a 5 años, con valores de 15,0 y 5,5\%, respectivamente, mientras que el $73,8 \%$ se encuentran en el rango de normal.
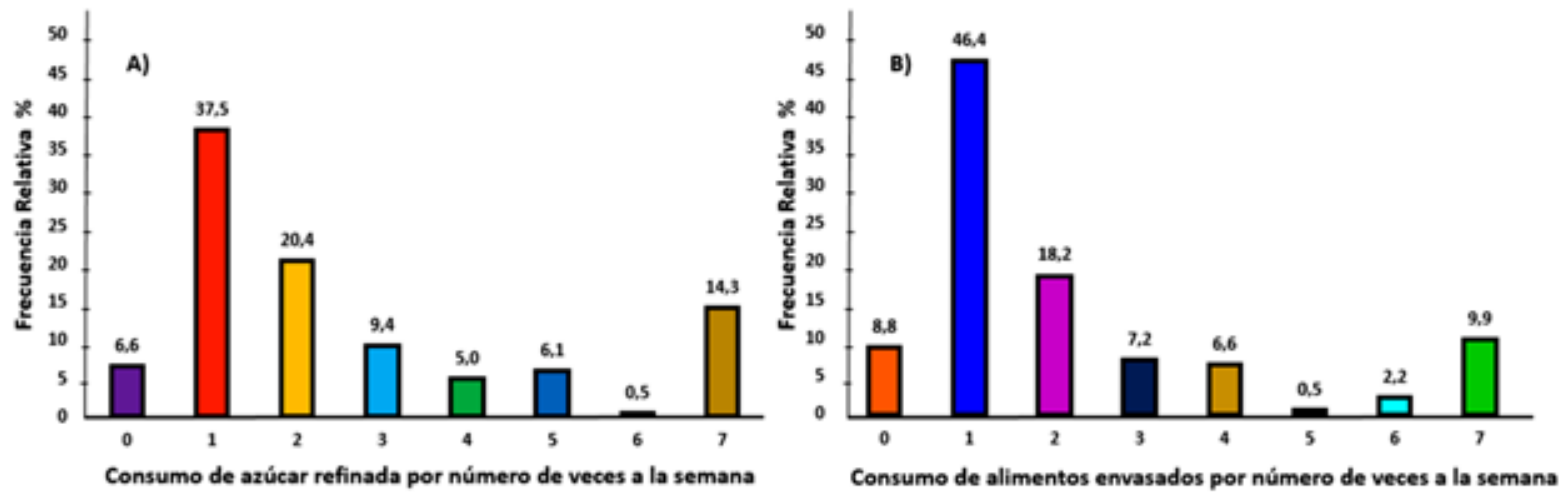

Figura 1: Frecuencia alimentaria en estudiantes de enseñanza básica de primer y segundo ciclo de 10 establecimientos educacionales de la comuna de Llanquihue $(n=181)$. Frecuencia del 1 al 7 (días de la semana) con respecto al consumo de A) azúcar refinada y B) Productos envasado.

Tabla 2. Parámetros estadísticos y límites de los intervalos de confianza de comparación de medianas apara menores y mayores a 5 años de las distintas escuelas que participaron en esta evaluación.

Parámetros estadísticos

Test Mood mediana

\begin{tabular}{|lcccccccc|}
\hline Ciclo & $\mathbf{n}$ & $\begin{array}{c}\text { Promedio } \\
\text { IMC }\end{array}$ & $\begin{array}{c}\text { DE } \\
\text { IMC }\end{array}$ & $\begin{array}{c}\text { Mínimo valor } \\
\text { IMC }\end{array}$ & $\begin{array}{c}\text { Máximo valor } \\
\text { IMC }\end{array}$ & $\begin{array}{c}\text { Mediana } \\
\text { IMC }\end{array}$ & $\begin{array}{c}\mathbf{9 5 , 0} \% \\
\text { Límite bajo }\end{array}$ & $\begin{array}{r}\mathbf{9 5 , 0} \% \\
\text { Límite alto }\end{array}$ \\
\hline Menor a 5 & 919 & 20,40 & 4,42 & 9,99 & 39,94 & 19,50 & 19,24 & 19,85 \\
Mayor a 5 & 126 & 17,50 & 2,62 & 10,71 & 30,20 & 16,92 & 16,64 & 17,31 \\
\hline
\end{tabular}




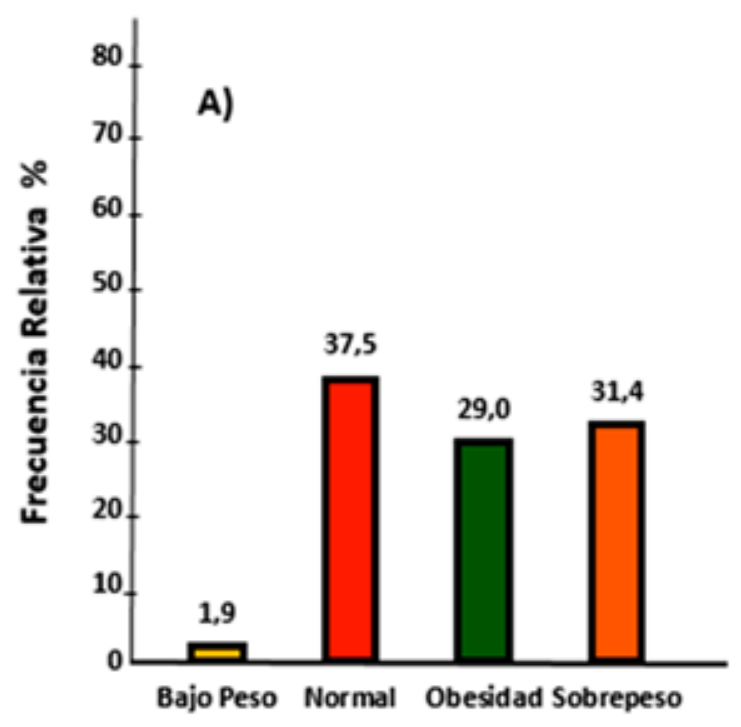

$$
\begin{gathered}
\text { Diagnóstico nutricional de niños } \\
\text { mayores de } 5 \text { años }
\end{gathered}
$$

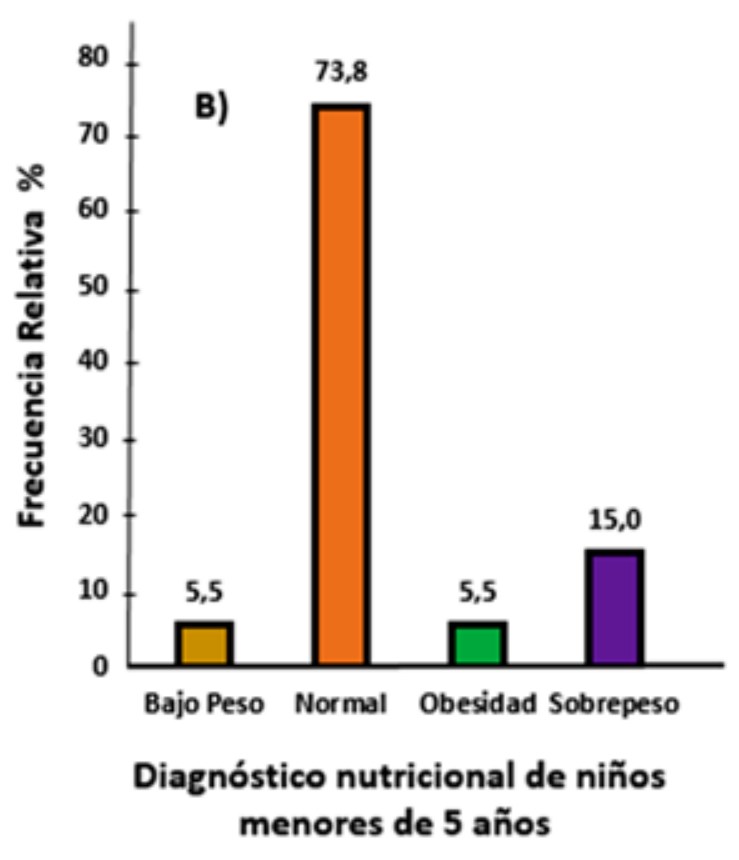

Figura 2: Diagnóstico nutricional de estudiantes pertenecientes a 5 establecimientos de la comuna de Llanquihue. A) niños mayores a 5 años $(n=919)$. B) niños menores a 5 años $(n=126)$.

\section{DISCUSIÓN}

El programa de Alimentación Escolar (PAE) tiene como finalidad entregar diariamente servicios de alimentación (desayuno, almuerzos, once, colaciones y cenas según corresponda), a los alumnos y alumnas en condición de vulnerabilidad de Establecimientos Municipales y Particulares Subvencionados del país durante el año lectivo adscritos al PAE, en los niveles de Educación prebásica, básica, media y adultos con el objetivo de mejorar su asistencia a clases y contribuir a evitar la deserción escolar. (https://pae.junaeb.cl/). En la comuna de Llanquihue y según lo observado en la encuestas realizadas, no todos los alumnos poseen este beneficio, por lo que los hábitos de alimentación son dispar, hay niños que pueden realizar todas sus comidas, y otros que no se alimentan hasta el almuerzo o la tarde al llegar a casa, al no dar importancia real a los hábitos de alimentos.

En relación al el ítem alimentación, se pueden observar que más del $60 \%$ de los niños encuestados almuerzan en el colegio, la misma cantidad de niños afirman que si consumen colaciones saludables entre comidas, las cuales son bajas o libres de azucares refinada y grasas saturadas, de bajo aporte calórico, aunque un número significativo de estudiantes, el 33,1\% menciona que no consumen colaciones saludables. El $15,7 \%$ de los niños almuerza dos veces tanto en la casa como en el colegio. Al respecto del ítem de frecuencia alimentaria (Figura 1A), el 37,5\% de los encuestados consumen algún alimentos con azúcar refinada al menos 1 vez a la semana y un número no menor, el $14,3 \%$ consumen este tipo de alimentos al menos 7 veces por semana lo cual señala la preferencia de los niños por alimentos altos en azucares, estos resultados pueden ser influenciados por la amplia oferta de productos poco saludables en el mercado y su bajo costo, como por el poco apoyo familiar, expresado en la falta de motivación para preparar comida saludable en el hogar ${ }^{27}$, esto fue corroborado por Olivares et al, en un estudio donde los padres señalaron preferir la comida «chatarra» y creer que a los niños no les gustan los alimentos saludables ${ }^{28}$. Sin embargo, existen intervenciones cortas que han producido cambios positivos en estado nutricional, conocimiento y consumo de los niños en Chile 29,30,31. $^{2}$.

La mayor parte de los encuestados consumen productos envasados (Figura 2B) al menos una (46,4\%) o 
dos veces por semana $(18,2 \%)$ y un porcentaje importante $(9,9 \%)$ consume estos tipos de alimentos al menos 7 veces por semana, por otro lado, respecto al consumo de comida chatarra entre los niños encuestados, el $47,1 \%$ consume comida chatarra al menos 1 vez por semana y un $17,1 \%$ al menos 2 veces por semana, siendo este tipo de comida muy poco saludables y altamente calóricas principalmente por presencia de grasas saturadas. Un estudio realizado en Talca con 1.401 estudiantes de cuarto año básico, demostró que no existía un adecuado conocimiento de hábitos y alimentación saludable, el 75\% no logró superar el rango de conocimientos esperados en esta materia ${ }^{32}$. Estilos de vida poco saludables asociados con una mayor ingesta de grasas, azúcar, baja actividad física y desequilibrio calórico, han demostrado promover el desarrollo de enfermedades cardiovasculares y metabólicas ${ }^{33}$. Existen evidencias que indican que el patrón de conducta alimentaria familiar tiene un papel relevante en el comportamiento de alimentación del niño ${ }^{33}$. Entender la susceptibilidad que lleva a algunas personas a subir de peso necesita el estudio de los factores fisiológicos, sociales y cognitivos que modulan la ingesta y el gasto ${ }^{34}$.

Del mismo modo, se pudo observar que el 58,5\% de los encuestados no realiza ningún tipo de actividad exprogramática o taller relacionado con el deporte al finalizar su jornada de estudios. Diversos estudios realizados en niños chilenos, han observado una escasa actividad física, asociada a un elevado número de horas frente al televisor, computador o en otras actividades sedentarias ${ }^{28}$, Olivares et al, concluyó que la proporción de niños que no realizaba actividad física fuera del colegio alcanzaba al 9,5 y 13,9\% en los niños y niñas de 8 a 9 años y al 6,9 y $17,7 \%$ en niños y niñas de 10 a 11 años, respectivamente ${ }^{35}$, estos resultados con bajos comparados a los obtenidos en este estudio, esta elevada proporción de niños sedentarios coincide con los resultados de los grupos focales ${ }^{28}$, donde niños y niñas manifestaron tener pocas motivaciones y una gran cantidad de barreras para realizar actividad física. Estudios señalan que la implementación de estrategias que combinan educación nutricional y realización de actividad física en escuelas por períodos de 4 a 12 meses, ayudan a mantener o mejorar levemente el IMC $\mathrm{IM}^{36,37}$. Es relevante aclarar que de las 5 escuelas participantes tan solo una de ellas presenta 4 horas pedagógicas de actividad física semanal, el resto dedica dos horas semanales, lo que puede validar la obtención de estos resultados.

Los resultados obtenidos en la comuna de Llanquihue son congruentes con los reportes anuales del Ministerio de Salud en Chile y con los resultados obtenidos en otros estudios descriptivos nacionales ${ }^{24,36,38}$ y del mismo modo se complementan con el diagnóstico del estado nutricional en donde se evidenció que el $55,6 \%$ de los niños encuestados padecen de sobrepeso u obesidad, un estudio realizado en España, afirma la prevalencia de sobrepeso en un $26 \%$ y la de obesidad, del $12,6 \%$ en menores de 8 a 17 años, 4 de cada 10 jóvenes sufren exceso de peso ${ }^{39}$.

Los datos obtenidos en el diagnóstico del estado nutricional (Figura 2A) de estudiantes mayores de 5 años $(n=919)$ de 5 establecimientos educacionales de la comuna de Llanquihue muestran que un $29,0 \%$ de niños se encuentran en condición de obesidad y un $31,4 \%$ con sobrepeso; un porcentaje bajo (1,9\%) se encuentra en bajo peso y el $37,5 \%$ se encuentra en un peso normal. Varios estudios demuestran que la obesidad infantil ha aumentado notablemente en los últimos décadas en prácticamente todos los países ${ }^{39,40}$, sin embargo, en Chile, la obesidad infantil se ha triplicado en las últimas décadas y continúa aumentando. Actualmente, el $25 \%$ de los niños de 6 años son obesos y el 30\% tienen sobrepeso por lo que es uno de los países con las tasas de obesidad infantil más altas del mundo ${ }^{16}$, lo que conduce a un panorama para nada alentador respecto a la nutrición de los niños no solo en la comuna de Llanquihue si no a nivel regional, nacional e incluso a nivel global.

\section{CONCLUSIÓN}

Los resultados de este estudio demuestran que los estudiantes de 4 a 13 años de edad en la comuna de Llanquihue califican en estado nutricional de sobrepeso, el 58,6\%. Los niños que participaron en este estudio no realizan actividad física extra programática y sus hábitos de consumo no favorecen esta condición. Este estudio sirve como base para el diseño y aplicación de estrategias de intervención educativas, que por medio de herramientas innovadoras y tecnológicas, contribuyan a educar en éste ámbito a niños(as) y jóvenes, esperando de este modo contribuir y generar cambios en la salud de los estudiantes de la comuna.

Agradecimientos: Este estudio fue financiado por el proyecto CORFO 17IS10-83162 de Innovación Social 2018. Agradecimientos a establecimientos educacionales que participaron en este estudio, quienes con su disposición y entusiasmo hicieron posible este proyecto, al CESFAM Los volcanes e Ilustre Municipalidad de Llanquihue por su colaboración en la realización de este estudio.

Conflicto de interes: Los autores no declaran conflicto de interés.

\section{BIBLIOGRAFÍA}

1. WHO - World Health Organization [web]. [Cited 18 December 2019] https://www.who.int/topics/obesity/es/

2. Lake A, Townshend T. Obesogenic environments: exploring the built and food environments. I R Soc Promot Health. 2006; 126: 262-267.

3. Calderón C, Forns $M$, Varea V. Childhood obesity: anxiety and cognitive and behavioral symptoms typical of eating disorders. An Pediatr. 2009; 71: 489-494. 
4. WHO - World Health Organization [web]. World health assembly. Report of the Commission to end childhood obesity Report of the Director General. [Cited 31 July 2019] available in: https://www.who.int/end-childhoodobesity/es/

5. Mussini, Micaela and Temporelli, Karina L. O. Obesity: a challenge for public policies. Estud Soc. 2013; 21(41): 165-184.

6. MINSAL - Ministry of Health. [web]. National food and nutrition policy. Ministry of Health. Santiago. Chile. First Ed. [updated 2017; cited 25 July 2019] available in: http:// www.bibliotecaminsal.cl/wp/wp-content/uploads/2018/01/ BVS_POL\%C3\%8DTICA-DE-ALIMENTACI\%C3\%93N-YNUTRICI\%C3\%93N.pdf

7. Cienfuegos E. 75 years ago The fight against child malnutrition. Chil Pediatr J. 2007; 78: 542-548.

8. FAO - Food and Drug Administration [web]. FAO / PAHO Nutrition Report: Chilean women lead obesity index in South America. [updated 2017; cited 14 July 2019] Available in: http://www.fao.org/chile/noticias/detailevents/es/c/1042961/

9. Moreno, G. M. Definition and classification of obesity. Rev Méd Clínica Las Condes. 2012; 23: 124-128.

10. Ariza C, Ortega-Rodríguez E, Sánchez-Martínez $F$, Valmayor S, Juárez O, Pasarín MI \& del Proyecto POIBA, G. D. I. The prevention of childhood obesity from a community perspective. Primary Care J. 2015; 47(4): 246-255.

11. Whitaker R, Wright J, Pepe M, Seidel K, Dietz W. Predicting obesity in young adulthood from childhood and parental obesity. New Eng J of Med. 1997; 337: 869-873.

12. OCDE-Organization for Economic Cooperation and Development. OECD Studies on Public Health Chile. "Towards a Healthier Future". Evaluation and recommendations. Ministry of Health, Government of CHILE. [updated 2019; cited

14 July 2019] Available in: https://www.oecd.org/ health/health-systems/Revisi\%C3\%B3n-OCDE-deSalud-P\%C3\%BAblica-Chile-Evaluaci\%C3\%B3n-yrecomendaciones.pdf

13. Estrada-Velasco B, Cruz M, García-Mena J, Salgado A, Romero J, Serrano $M$, et al. Childhood obesity is associated to the interaction between firmicutes and high energy food consumption. Nut Hosp J. 2015; 31: 1074-1081.

14. RSA - Sanitary regulations of food N $977 / 96$ of Ministry of Health, Government of CHILE. [updated 2019; cited 14 July 2019] Available in: https://www.minsal.cl/sites/ default/files/files/DECRETO_977_96\%20actualizado\%20 a\%20Enero\%202015(1).pdf

15. Vio del Rio F. Increase in obesity in Chile and in the world. Rev Chil Nutr. 2018; 45: 1.

16. IUNAEB-National Board of School Assistance and Scholarships of Government of CHILE. [updated 2019; cited 14 July 2019] Available in: https://www.junaeb.cl/ mapa-nutricional

17. Nuño $M$, Hevia $M$, Bustos $C$, Florenzano $R$, Fritsch $R$. Distortion of body image in mothers towards their overweight or obese children. Rev Chil Nutr. 2017; 44(1): 28-32.

18. Herrera J, Lira M, Kain J. Socio-economic vulnerability and obesity in Chilean primary schoolchildren: comparison between 2009 and 2013. Rev Chil Pediatr. 2017; 88: 736-743.

19. Raimann T. X. Obesidad y sus complicaciones. Rev Méd Clínica Las Condes. 2011; 22: 20-26.
20. Gutiérrez Valverde JM, Guevara Valtier MC, Enríquez Reyna MC, Morales P, de los Ángeles M, del Ángel $H$, Landeros Olvera E. Study in families: environmental and cultural factors associated with overweight and obesity. Global Nursing J. 2017; 16; 1-19.

21. Bustos N, Albala C. Overview of programs in the world on control and prevention of obesity. In Mardones, $F$ (editor). Obesity in Chile. What can we do? 2018. Ediciones UC (segunda edición).

22. Goldstein B. Eduardo. Julio Parliamentary Technical Advisory Policies against obesity in Chile: Acknowledgments and shortcomings. Library of the National Congress of Chile Parliamentary Technical Advisory

23. Lera L, Fretes G, Gonzalez C, Salinas J, Vio F. Validation of an instrument to assess consumption, habits and feeding practices in school children aged 8 to 11 years. Nutr Hosp. 2015; 31: 1997-1988.

24. Burrows R, Diaz E, Sciaraffia M, Gattas V, Montoya A, Lera L. Habits of ingestion and physical activity in schoolchildren, according to the type of establishment they attend. Rev Med Chile. 2008; 136: 53-63.

25. Manzini J. Declaration of Helsinki: ethical principles for medical research on human subjects. Bioethic Act. 2000; 6: 321-334.

26. Lacobucci D, Duhachek A. Advancing alpha: Measuring reliability with confidence. JCP 2003; 13: 478-487.

27. Macias AI, Gordillo LG, Camacho EJ. Eating habits in school-age children and the health education paper. Rev Chil Nutr. 2012; 39(3): 40-43.

28. Olivares S, Bustos N, Moreno X, Lera L, Cortez S. Food and physical activity attitudes and practices in obese children and their mothers in Santiago, Chile. Rev Chil Nutr. 2006; 33: 170-179.

29. Vio F, Salinas J, Montenegro E, González CG, Lera L. Impact of a nutrition education intervention in teachers, preschool and basic school-age children in valparaiso region in Chile. Nutr Hosp. 2014; 29: 1298-1304.

30. Ratner R, Durán S, Garrido MJ, Balmaceda S, Jadue L, Atalah E. Impact of an intervention on diet and physical activity on obesity prevalence in schoolchildren. Nutr Hosp. 2013; 28: 1508-1514.

31. Salinas J, González CG, Fretes G, Montenegro E. Theoretical and methodological basis for a healthy food educational program in Chilean basic schools. Rev Chil Nutr. 2014; 41(4): 343-350.

32. Pino J. Food-nutritional knowledge and nutritional status of fourth-year students according to private and subsidized establishments in the city of Talca. Rev Chil Nutr. 2010; 37: 4.

33. Vasquez, F., Corvalan, C., Uauy, R., \& Kain, J. Impact of gaining or maintaining excessive weight in infancy on markers of metabolic homeostasis in young children: A longitudinal study in Chilean children. Prev Med Rep. 2018; 12: 298-303.

34. Errandonea, U. I. Obesity and eating disorders. Rev Méd Clínica Las Condes. 2012; 23: 165-171.

35. Olivares S, Kain J, Lera L, Pizarro F, Vio F, Morón C. Nutritional status, food consumption and physical activity among Chilean school children: a descriptive study. Eur J Clin Nutr. 2004; 58: 1278-1285.

36. Lobos Fernández L, Leyton Dinamarca B, Kain Bercovich J, Vio del Río F. Evaluation of an educational intervention for the prevention of childhood obesity in basic schools 
in Chile. Nut Hosp J. 2013; 28: 1156-1164.

37. Aguilar Cordero MJ, González Jiménez E, García García C), García López PA, Álvarez Ferre J, Padilla López CA, et al. Obesity in a school children population from Granada: assessment of the efficacy of an educational intervention. Nutr Hosp. 2011; 26: 636-641.

38. Davis S, Myers O, Cruz T, Morshed A, Canaca G, Keane $P, O^{\prime}$ Donald E. Chile: Outcomes of a group randomized controlled trial of an intervention to prevent obesity in preschool Hispanic and American Indian children. Prev Med J. 2016; 89: 162-168.

39. Sánchez-Cruz J J, Jiménez-Moleón J J, Fernández-Quesada F, Sánchez M J. Prevalence of childhood and youth obesity in Spain in 2012. Rev Esp Car. 2013; 66: 371-376.

40. Jacoby E, Grajeda R, Contreras A, Hospedales J. The epidemic of childhood obesity in the Americas must be stopped: governmental and PAHO leadership are crucial. Int J Obes. 2013; 3(Suppl 1): s15-s17. 